\title{
Hypertrophic pulmonary osteoarthropathy on bone scintigraphy and somatostatin receptor scintigraphy
}

Georgios Meristoudis ${ }^{1}$, Ioannis llias², Vasilios Giannakopoulos ${ }^{3}$

\section{Dear Editor,}

Hypertrophic osteoarthropathy (HOA) as a paraneoplastic disorder is most often associated with pulmonary malignancies ${ }^{1}$. Bone scintigraphy (BS) is known to be useful for detecting $\mathrm{HOA}^{1,2}$. Here, we present a lung cancer patient who demonstrated findings consistent with HOA on BS and somatostatin receptor scintigraphy (SRS). To the best of our knowledge, this is the first report of HOA visualized by SRS.

A female aged 67 years, smoker (47 pack-years), presented with a 3-month history of generalized arthralgia, painful edema of the limps, and finger clubbing. BS using ${ }^{99 \mathrm{~m} T c-M D P}$ demonstrated increased linear periosteal uptake in the long bones of the legs (Figure $1 \mathrm{~A}$ ), a characteristic scintigraphic pattern of HOA. A chest X-ray was done (given her history of smoking) and showed a mass in the right posterior hemithorax confirmed by computed tomography (CT) scan (Figure 2C). Further functional imaging with SRS using ${ }^{99 \mathrm{~m} T c}$-octreotide revealed that the pulmonary lesion was positive for somatostatin receptors (Figure 2D). Also, it showed mildly increased tracer uptake along the periosteum of both lower extremities (Figure 1B). Ultimately, histopathological examination revealed lung adenocarcinoma.

$\mathrm{HOA}$, also named Marie-Bamberger syndrome was first described in the 1890 s and is characterized clinically by periostitis of tubular bones, digital clubbing, and arthritic symptoms ${ }^{1}$. It can be classified as primary (very rare) or secondary (approximately 95\% to 97\% of cases). Secondary HOA is associated with a wide spectrum of diseases, including a variety of pulmonary disorders, also known as 'hypertrophic pulmonary osteoarthropathy' (e.g. primary and metastatic lung cancer, lung abscess, tuberculosis, sarcoidosis, emphysema, bronchiectasis, pulmonary fibrosis, and mesothelioma), cardiovascular disorders (e.g. cyanotic congenital heart disease, infective endocarditis), gastrointestinal disorders (inflammatory bowel disease and hepatic cirrhosis) and various other disorders. Secondary HOA is more frequently related to pulmonary malignancies (in up to 90\%), especially lung cancer ${ }^{1}$. According to published reports, $4 \%$ to $32 \%$ of lung cancer patients develop $\mathrm{HOA}^{3}$. The clinical manifestations of HOA may precede the diagnosis of the underlying disease. BS is a sensitive imaging modality for evaluating a wide variety of skeletal disorders, including $\mathrm{HOA}^{1,2}$. This modality has higher sensitivity for detecting bone metastases in patients with lung cancer compared to SRS ${ }^{4,5}$. The exact mechanism of pathogenesis of HOA and clubbing remains unknown. Two models have been proposed: a neurogenic pathway and a humoral pathway. In the neurogenic pathway, diseased organs innervated by the vagus nerve induce a neural reflex leading to vasodilatation and increased blood flow to the extremities ${ }^{6}$. In the humoral pathway cytokines and growth factors (platelet-derived growth factor, prostaglandin $\mathrm{E}$, and vascular endothelial growth factor) induce connective tissue and periosteal proliferation ${ }^{1}$.

Our case report highlights the usefulness of BS in detecting HOA and the importance of additional (anatomical and functional) chest imaging in the evaluation of malignancy-related HOA. Additionally, this case report shows that SRS can identify HOA, which is a new addition to the literature regarding the range of potential scintigraphic imaging of this syndrome. Moreover, it lends credence to the therapeutic option of octreotide (a synthetic analogue of

\author{
AFFILIATION \\ 1 Department of Nuclear Medicine, \\ Hippokration General Hospital, Thessaloniki, \\ Greece \\ 2 Department of Endocrinology, Diabetes \\ and Metabolism, Elena Venizelou Hospital, \\ Athens, Greece \\ 3 Department of Nuclear Medicine, General \\ Hospital of Thoracic Diseases Sotiria, Athens, \\ Greece
}

\section{CORRESPONDENCE TO}

Georgios Meristoudis. Department of Nuclear Medicine, Hippokration General Hospital, Konstantinoupoleos 49, 54642, Thessaloniki, Greece. E-mail: meristoudis@yahoo.gr ORCID ID: https://orcid.org/0000-0002-03826962

\section{KEYWORDS}

hypertrophic osteoarthropathy, lung cancer, periostitis, bone scintigraphy, somatostatin receptor scintigraphy

Received: 26 April 2021

Revised: 16 August 2021

Accepted: 25 August 2021 
Figure 1. A. BS images show bilateral, linear uptake of the radiopharmaceutical in the femurs and tibia (arrows), findings compatible with the tram line or double stripe sign, and to a lesser degree uptake in the distal upper extremities, including the terminal phalanges (dotted arrows). B. SRS images reveal mildly increased linear uptake, more pronounced along the distal femoral and tibial metaphyses (arrows).

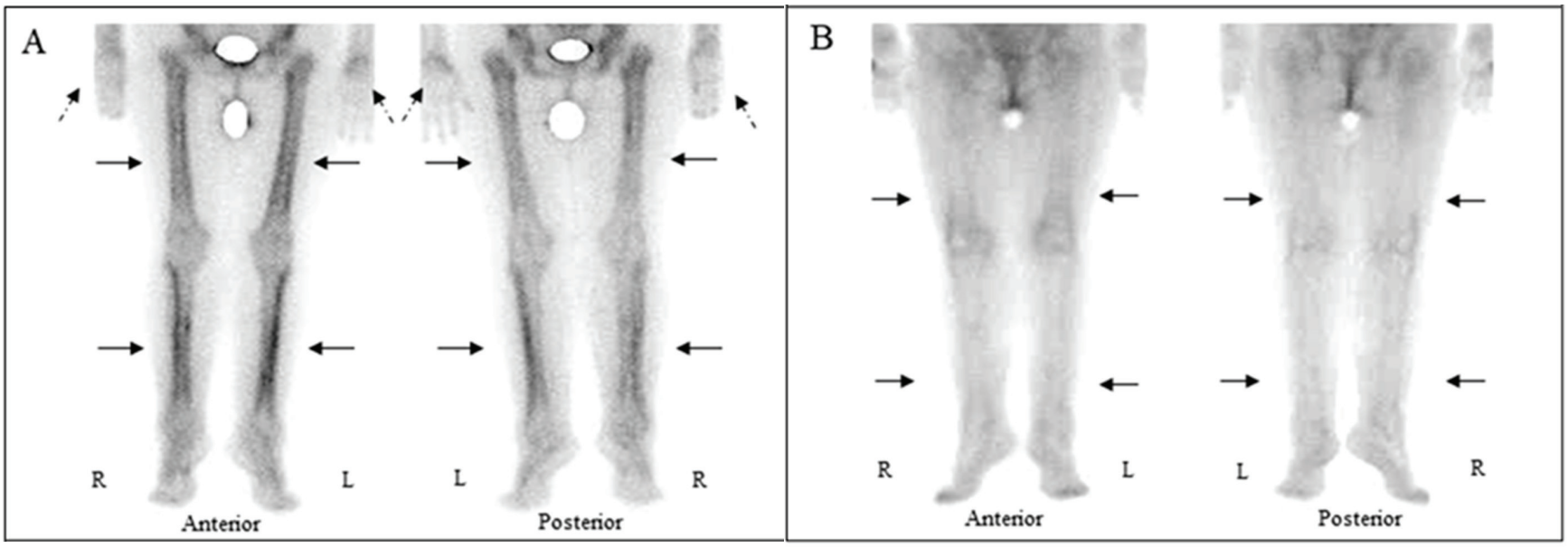

Figure 2. C. CT scan of the chest showing a mass posteriorly in the upper right hemithorax (white arrow). D. Planar SRS anterior image of the chest demonstrating increased tracer activity corresponding to the lesion visualized on the CT scan (black arrow). Note the physiological intense uptake in the liver and spleen.
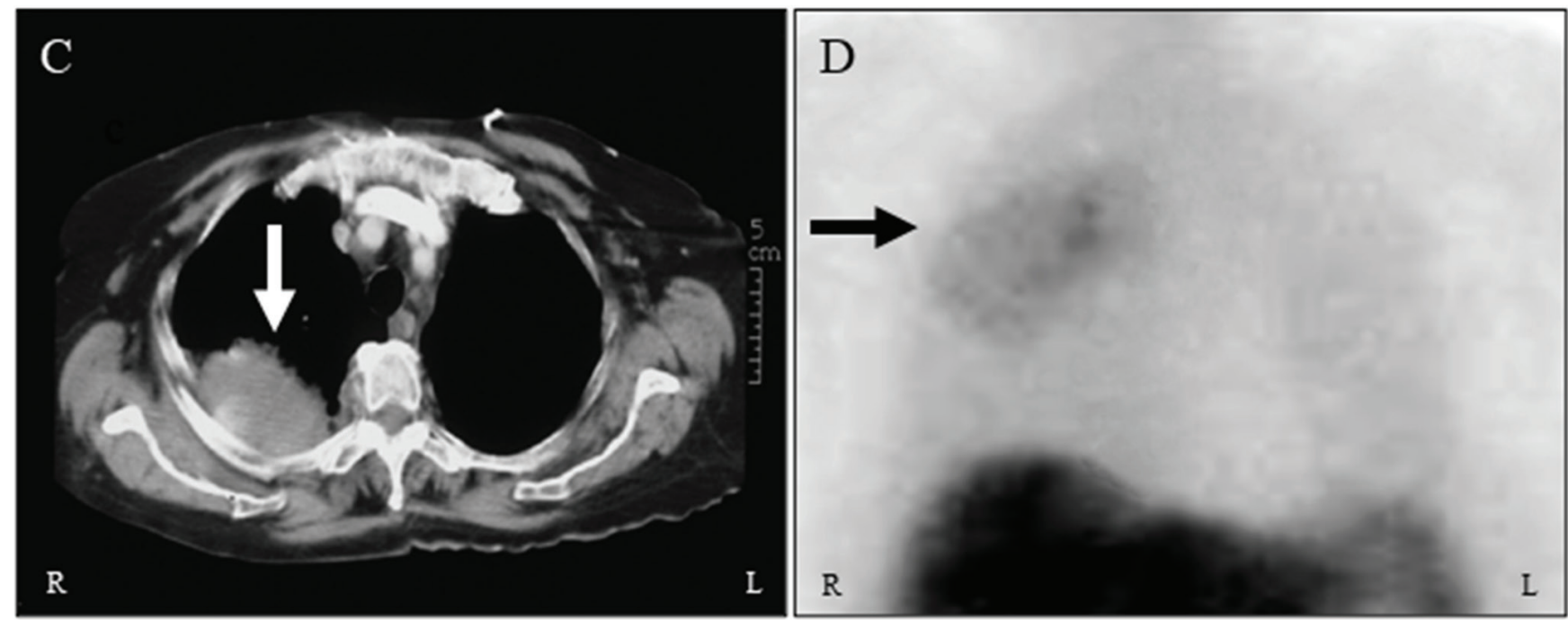

somatostatin) for symptomatic treatment of $\mathrm{HOA}^{3,7-10}$.

\section{CONFLICTS OF INTEREST}

The authors have completed and submitted the ICMJE Form for Disclosure of Potential Conflicts of Interest and none was reported.

\section{FUNDING}

There was no source of funding for this research.

\section{ETHICAL APPROVAL AND INFORMED CONSENT}

Ethical approval was not required for this study.

\section{DATA AVAILABILITY}

The data supporting this research cannot be made available for privacy reasons. 
PROVENANCE AND PEER REVIEW

Not commissioned; internally peer reviewed.

\section{REFERENCES}

1. Yap FY, Skalski MR, Patel DB, et al. Hypertrophic Osteoarthropathy: Clinical and Imaging Features. Radiographics. 2017;37(1):157-195. doi:10.1148/rg.2017160052

2. Qian X, Qin J. Hypertrophic pulmonary osteoarthropathy with primary lung cancer. Oncol Lett. 2014;7(6):2079-2082. doi:10.3892/ol.2014.2022

3. Nguyen S, Hojjati M. Review of current therapies for secondary hypertrophic pulmonary osteoarthropathy. Clin Rheumatol. 2011;30(1):7-13. doi:10.1007/s10067-010-1563-7

4. Meristoudis G, Ilias I, Batsakis C, Christakopoulou J. Bone

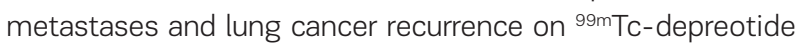
imaging. Hell J Nucl Med. 2008;11(3):185-186. Accessed August 25, 2021. https://www.nuclmed.gr/wp/wp-content/ uploads/2017/03/41-2.pdf

5. Meristoudis G, Ilias I, Giannakopoulos V, Batsakis C, Christakopoulou J. Discrepant uptake between ${ }^{111} \mathrm{In}$ pentetreotide and ${ }^{99 m} \mathrm{Tc}-\mathrm{MDP}$ in bone metastases of smallcell lung cancer. Hell J Nucl Med. 2009;12(3):283-284. Accessed August 25, 2021. https://www.nuclmed.gr/wp/ wp-content/uploads/2017/03/36.pdf

6. Treasure T. Hypertrophic pulmonary osteoarthropathy and the vagus nerve: an historical note. J R Soc Med. 2006;99(8):388-390. doi:10.1258/jrsm.99.8.388

7. Birch E, Jenkins D, Noble S. Treatment of painful hypertrophic osteoarthropathy associated with non-small cell lung cancer with octreotide: a case report and review of the literature. BMJ Support Palliat Care. 2011;1(2):189-192. doi:10.1136/bmjspcare-2011-000052

8. Penson RT, Rudd RM. Commentary: octreotide and hypertrophic pulmonary osteoarthropathy. Thorax. 1997;52(3):297-298. doi:10.1136/thx.52.3.297

9. Johnson SA, Spiller PA, Faull CM. Treatment of resistant pain in hypertrophic pulmonary osteoarthropathy with subcutaneous octreotide. Thorax. 1997;52(3):298-299. doi:10.1136/thx.52.3.298

10. Angel-Moreno Maroto A, Martínez-Quintana E, SuárezCastellano L, Pérez-Arellano JL. Painful hypertrophic osteoarthropathy successfully treated with octreotide. The pathogenetic role of vascular endothelial growth factor (VEGF). Rheumatology (Oxford). 2005;44(10):1326-1327. doi:10.1093/rheumatology/keh720 